\title{
Research on Multi-target Scales Estimation Based on the Theory of Fuzzy Clustering
}

\author{
Wang Bai-he ${ }^{1,}$, Liu Dai-zhi ${ }^{1}$, Huang Shi-qi ${ }^{1}$ \\ ${ }^{1}$ Xi'an H-tech Research Institute, Xi'an, 710025, China \\ aLilywbh@sina.com
}

Keywords: Scale recognition; Target identification; Multi-target system; Fuzzy clustering

\begin{abstract}
Scale recognition is the effective technology in underwater target identification. The existing methods can only solve the problem of single-target scale estimation and are not adaptable to the problem of multi-target scale estimation and recognition in complex counterwork. A method named FC based on fuzzy clustering analysis was put forward to analyze and estimate the multi-target scales in this paper. In the method, all the highlights collected by homing system were analyzed by dynamic fuzzy clustering with the principle of shortest distance, and scales were estimated separately based on the lights belonged to deferent sorts. Simulation results show that the problem of multi-target scale recognition can be solved effectively with FC method.
\end{abstract}

\section{Introduction}

Multi-target system (MTS) is a new-style homing system to improve the performance of underwater vehicles in the complex counterwork ${ }^{[1]}$. In complex counterwork, targets will release some acoustic countermeasure equipments to disturb or beguile the underwater vehicle, and then multi-target will appear in the working sector of the vehicle. So underwater vehicles must be able to detect and track multi-target in the condition of complex counterwork, and multi-target detection, recognition and tracking are the key technologies in $\mathrm{MTS}^{[2]}$.

At present, scale recognition is the effective target identification technology applied in active homing system of underwater vehicles, and scale information of targets must be got at first. The methods of short impulse incision, short time cross-spectrum and high-resolution dimension estimation in common used can only solve the problem of single-target scale estimation ${ }^{[3-5]}$. But in MTS, the information of highlights belonged to deferent targets is mixed and can't be distinguished. So signal-target scale estimation methods usually used are not adaptable to multi-target scale estimation and recognition, and can't testify the advantage of MTS.

A method named FC based on fuzzy clustering analysis is put forward to analyze and estimate the multi-target scales in this paper. In the method, all the highlights collected by homing system are analyzed by dynamic fuzzy clustering with the principle of shortest distance, and dimensions are estimated separately based on the lights belonged to deferent sorts. Simulation results show that the method of FC can resolve the problem of multi-target scales estimation effectively.

\section{The theory of fuzzy clustering}

In science technology and Economics management, classifying is usually to do with some things according to a certain standard such as the similar degree and correlation. Classifying can be done with lights belonged to different targets according to the principle that the distance of lights belongs to same target must be smaller. The fuzzy clustering method is more realistic because of the fuzzy property of classifying in science technology and Economics management.

The usual steps of fuzzy clustering algorithm are as following ${ }^{[6]}$ :

(1) Data standardization

The discussing field of $U=\left\{x_{1}, x_{2}, \cdots, x_{m}\right\}$ is the object to be classified and every object contains $n$ indexes:

$$
x_{i}=\left\{x_{i 1}, x_{i 2}, \cdots, x_{i n}\right\}, \quad i=1,2, \cdots, m
$$


The original data matrix $R$ is expressed as

$$
R=\left[\begin{array}{cccc}
x_{11} & x_{12} & \cdots & x_{1 n} \\
x_{21} & x_{22} & \cdots & x_{2 n} \\
\cdots & \cdots & \cdots & \cdots \\
x_{m 1} & x_{m 2} & \cdots & x_{m n}
\end{array}\right]
$$

Data standardization is to wipe off the dimension of the data and compress it to $[0,1]$ to fill the demand of fuzzy matrix and it is realized as following:

(a) Standard deviation transformation

The expression of standard deviation transformation is defined as:

$$
x_{i k}^{\prime}=\frac{x_{i k}-\bar{x}_{k}}{s_{k}}, \quad i=1,2, \cdots, m ; k=1,2, \cdots, n
$$

where $\bar{x}_{k}=\frac{1}{m} \sum_{i=1}^{m} x_{i k}, s_{k}=\sqrt{\frac{1}{m} \sum_{i=1}^{m}\left(x_{i k}-\bar{x}_{k}\right)^{2}}$.

The mean is 0 and standard deviation is 1 of every variable after the transform and the influence of dimension is wiped off. But $x_{i k}^{\prime}$ is not within $[0,1]$ always yet, so further transform is needed.

(b) Hyper-deviation transformation

The expression of hyper-deviation transformation is defined as:

$$
x_{i k}^{\prime \prime}=\frac{x_{i k}^{\prime}-\min _{1 \leq i \leq m}\left\{x_{i k}^{\prime}\right\}}{\max _{1 \leq i \leq m}\left\{x_{i k}^{\prime}\right\}-\min _{1 \leq i \leq m}\left\{x_{i k}^{\prime}\right\}} \quad(k=1,2, \cdots, n)
$$

Obviously, $0 \leq x_{i k}^{\prime \prime} \leq 1$ and the influence of dimension is wiped off also.

(2) Demarcation (fuzzy-similar matrix setting)

The discussing field is defined as $U=\left\{x_{1}, x_{2}, \cdots, x_{m}\right\}, x_{i}=\left\{x_{i 1}, x_{i 2}, \cdots, x_{i n}\right\}$.Similarity coefficient and fuzzy-similar matrix are defined according to traditional clustering method. The similar degree of $x_{i}$ and $x_{j}$ is define as $r_{i j}=R\left(x_{i}, x_{j}\right)$ and $r_{i j}=R\left(x_{i}, x_{j}\right)$ is solved by the traditional methods of similarity coefficient, distance and the other methods.

(3) Clustering (dynamic clustering)

Direct clustering gets clustering diagram based on fuzzy-similar matrix. The steps of clustering are as following:

(a) Threshold is valued as $\lambda_{1}=1$ and similar class $\left[x_{i}\right]_{R}$ is set to every $x_{i}$ as:

$$
\left[x_{i}\right]_{R}=\left\{x_{j} \mid r_{i j}=1\right\}
$$

which puts $x_{i}$ and $x_{j}$ met $r_{i j}=1$ together to compose similar class. The deference between similar class and equivalent class is that deferent similar class may have common elements, which is that $\left[x_{i}\right]_{R}=\left\{x_{i}, x_{j}\right\},\left[x_{j}\right]_{R}=\left\{x_{j}, x_{k}\right\},\left[x_{i}\right] \cap\left[x_{j}\right] \neq \varnothing$ may happen. Similar classes with common elements may be combined to get equivalence classes on the level of $\lambda_{1}=1$.

(b) Threshold is valued as secondary value $\lambda_{2}$. The element pair $\left(x_{i}, x_{j}\right)$ with the similar degree $\lambda_{2} \quad\left(r_{i j}=\lambda_{2}\right)$ is found out and the corresponding equivalent classes contained $x_{i}$ and $x_{j}$ on the level of $\lambda_{1}=1$ are combined to get the equivalent classes corresponding to $\lambda_{2}$.

(4) The rest steps may be deduced by analogy until the number of the equivalent classes is equal to the setting value. 


\section{Multi-target scales estimation based on the method of FC}

According to the foregoing statement, multi-target scales estimation method (named FC method) based on the theory of fuzzy clustering is proposed in this paper. The specific steps are described as following:

(1) Data access. The delay and azimuth information of all the lights is accessed after multi-target detection and multi-parameter estimation based on the target echo information got by the homing system, which is noted as polar coordinate $\left\{R_{i}, \theta_{i}\right\}$. The corresponding position vector is defined as $P_{i}=R_{i} \exp \left(j \theta_{i}\right)$ and $i$ is the serial number of the lights.

(2) Distance calculation. The position of all the lights on the platform is confirmed based on the information of delay and azimuth accessed by step 1, which is used to calculate the Euclidean distance of every light pair as following formula:

$$
L=\left|P_{i}-P_{j}\right|=\sqrt{\left(R_{i} \cos \theta_{i}-R_{j} \cos \theta_{j}\right)^{2}+\left(R_{i} \sin \theta_{i}-R_{j} \sin \theta_{j}\right)^{2}}
$$

where $P_{i}$ and $P_{j}$ are the two lights in the pair.

(3) Fuzzy similar matrix calculation. The original data matrix of lights is constructed based on the distance parameter got by step 2, and the fuzzy similar matrix is got after standardization.

(4) Clustering. The maximum of fuzzy similar matrix is set as the initial threshold, direct clustering is done based on the information of light, and the number of classes is noted as $c_{i}$. Threshold $\lambda_{i}$ is reduced continuously until $c_{i}$ is equal to the number of targets detected.

(5) The multi-target scales calculation. The polar coordinate of nearest light is noted as $\left\{R_{1}, \theta_{1}\right\}$, and the polar coordinate of most far light is noted as $\left\{R_{d}, \theta_{d}\right\}$. The information of all the lights belonged to one class (or one target) is used to calculate the target scale according to the formula as following:

$$
L=(C / 2) \sqrt{\tau_{1}^{2}+\tau_{d}^{2}-2 \tau_{1} \tau_{d} \cos \left(\theta_{1}-\theta_{d}\right)}
$$

The estimation of distance $R_{i}$ is equaled to measure the delay $\tau_{i}$ between echo and emission signal. The parameter $\tau_{i}$ is defined as $\tau_{i}=2 R_{i} / C$ and $C$ is the velocity of underwater sound. The simulation flow chart of multi-target scales estimation of FC method is showed as Fig.1.

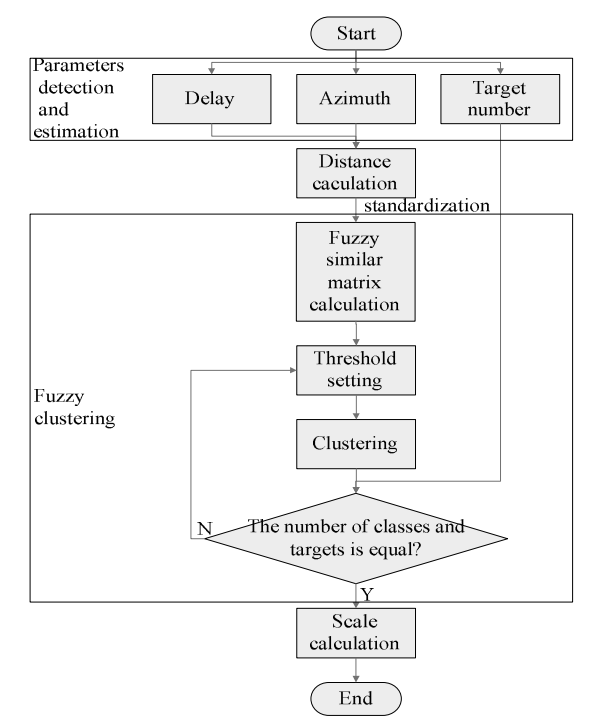

Fig. 1 The simulation flow chart of multi-target scales estimation based on FC method 


\section{Simulations}

In the whole working progress of homing system, after multi-target detection and parameter estimation based on echo signal, the position information of 44 lights belongs to 3 targets accessed is showed as Fig.2.

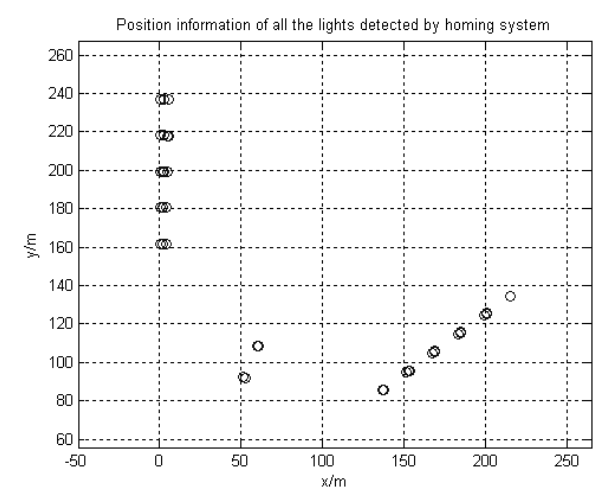

Fig.2 Position information of all the detected lights

The homing system can not classify the lights belonged to the same target and estimation the target scales because the information is mixed in a big data packet. FC method is applied. All the nearest lights are congregated to one class at first with the threshold is setting as 0.9993 , and 44 classes are got as a result. The threshold is reduced continuously and the number of class $c_{i}$ is reduced accordingly. The corresponding relation of the threshold $\lambda_{i}$ and the number of class $c_{i}$ is showed as Tab.1.

Table 1 Corresponding relation of the threshold $\lambda_{i}$ and the class number $c_{i}$

\begin{tabular}{|c|c|c|c|c|c|c|c|c|c|}
\hline$\lambda_{i} 0.9993$ & 0.9992 & 0.9991 & 0.9990 & 0.9989 & 0.9963 & 0.9929 & 0.9926 & 0.9924 & 0.9921 \\
\hline$c_{i} 44$ & 38 & 34 & 31 & 29 & 28 & 27 & 26 & 25 & 24 \\
\hline$\lambda_{i} 0.9916$ & 0.9915 & 0.9913 & 0.9906 & 0.9905 & 0.9897 & 0.9896 & 0.9888 & 0.9296 & 0.9212 \\
\hline$c_{i} 20$ & 19 & 18 & 17 & 16 & 15 & 14 & 13 & 8 & 3 \\
\hline
\end{tabular}

When the threshold is reduced to 0.9897 , all the lights are congregated to 15 classes. The simulation result is showed as Fig.3. When the threshold is reduced to 0.9296, all the lights are congregated to 8 classes. The simulation result is showed as Fig. 4.

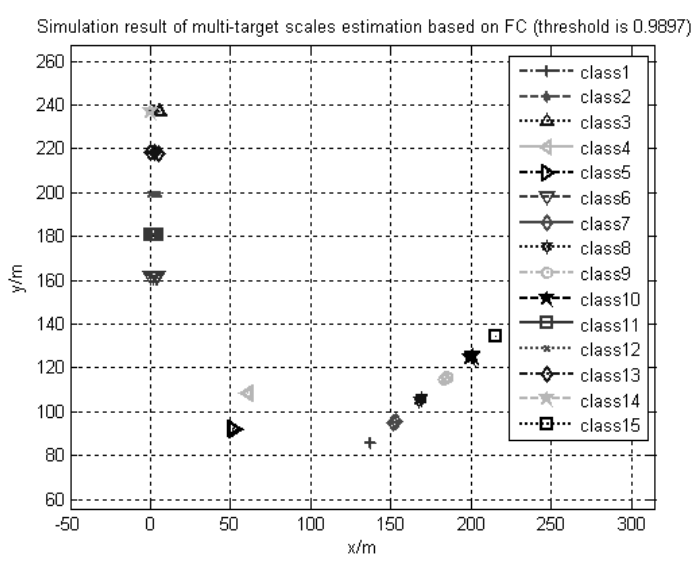

Fig.3 Simulation result (threshold is 0.9897)

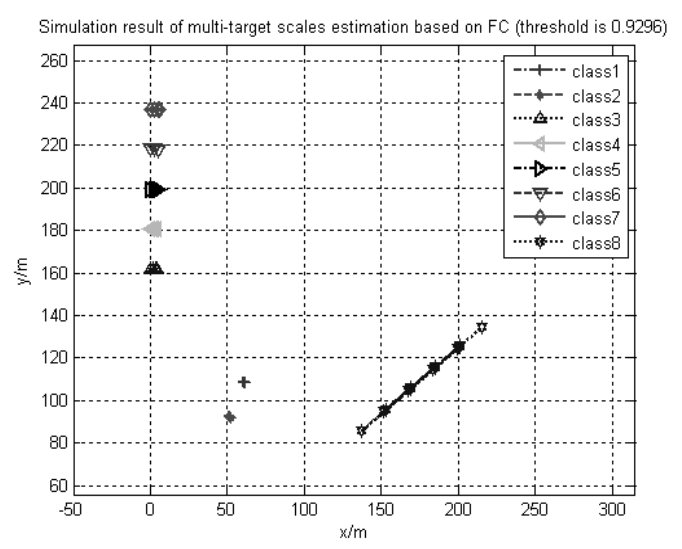

Fig.4 Simulation result (threshold is 0.9296) 
When the threshold is reduced to 0.9212 , all the lights are congregated to 3 classes. The simulation result is showed as Fig.5.

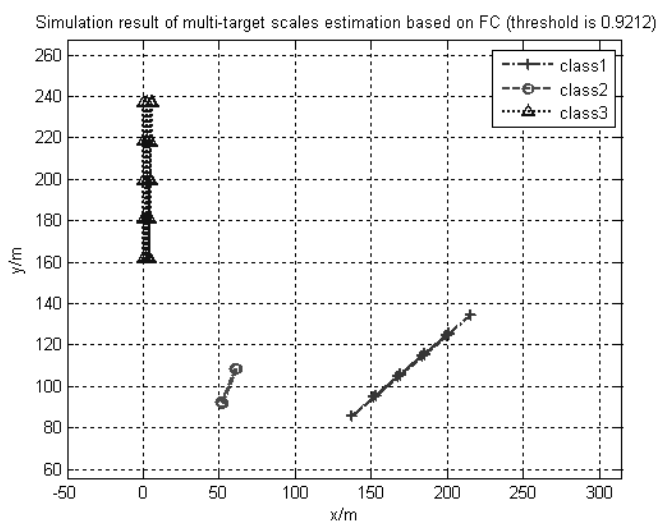

Fig.5 Simulation result (threshold is 0.9212)

Based on the foregoing simulation result, when the threshold is reduced to 0.9212, all the lights are congregated to 3 classes, which is equal to distribute all the lights into 3 targets. The lights belonged to one target are combined together with a line which is showed as Fig.5. Then single target scale estimation method is used to estimate the scale of every target. The scale of No.1 target is 91.7490 meters, the scale of No.2 target is 18.7803 meters, and the scale of No.3 target is 75.1436 meters. We can draw a conclusion that the scale of No.2 target is too small to be a real target and it is judged to be a false target. The FC method proposed in this paper can solve the problem of multi-target scales estimation effectively.

\section{Conclusions}

The FC method is proposed in this paper on the research of multi-target scales estimation after limit analysis of the method of single target scale estimation used in MTS. The theoretical model is established and experimental analysis is done in the paper. Simulation result shows that the FC method can solve the problem of multi-target scales estimation effectively, realizes the scale recognition of multi-target homing system and improve the tactical and technical performance of underwater vehicles.

Foundation item: Supported by the National Natural Science Foundation of China under Grant No. 60572098.

\section{References}

[1] Wang BS, Li FS. The Research on Multiple targets Tracking Based on the Data Fusion Technique. Jouranl of Xidian University (Natural Science), 1998, 25(3) 269-272.

[2] Tang Q, Huang JG, Yang XD. Study on Approaches for Track Initiation Based on Dynamic States. Acta Aeronautica at Astronautica Sinica, 2007, 28(2) 407-410.

[3] Yang B, Wang HL, Yang BM. The Study of Acoustic Imaging Vertical Dimension Identification Using High-resolution Direction-finding. Ship Science and Technology, 2010, 32(1) 76-78.

[4] Wang MZ, Hao CY, Huang XW. Target Dimension Recognition Based on Short Time Cross-Spectrum by Using Splitting Beam Array. Journal of Data Acquisition \& Processing, 2003, 18(4) 440-443.

[5] Liu ZH, Fu ZP, Wang MZ. Underwater Target Identification Based on the Methods of Bearing and Cross-spectrum. ACTA ARMAMENTARII, 2006, 27(5) 932-935.

[6] Xie JJ, Liu CP. Fuzzy Mathematic Method and the Application. Wuhan, 2000. 